Submitted to 1995 Particle Accelerator Conference,

Da11as, Texas, May 1-5, 1995

$\operatorname{Con} 8-9505 / 2--16$

BNL-61851

\title{
Microwave Measurements of the BNL/SLAC/UCLA 1.6 Cell Photocathode RF Gun*
}

\author{
D. T. Palmer, R. H. Miller and H. Winick \\ Stanford Linear Accelerator Center \\ Stanford University, Stanford CA 94309 \\ X.J. Wang, K. Batchelor, M. WoOdLe and I. Ben-ZVI \\ Brookhaven National Laboratory Accelerator Test Facility \\ Upton, NY 11973
}

\begin{abstract}
The longitudinal accelerating field $E_{z}$ has been measured as a function of azimuthal angle in the full cell of the cold test model for the 1.6 cell BNL/SLAC/UCLA \#3 S-band RF Gun using a needle rotation / frequency perturbation technique [1]. These measurements were conducted before and after symmetrizing the full cell with a vacuum pump out port and an adjustable short. Two different waveguide to full cell coupling schemes were studied. The dipole mode of the full cell is an order of magnitude less severe before symmetrization for the $\theta$-coupling scheme. The multi-pole contribution to the longitudinal field asymmetry are calculated using standard Fourier series techniques. The Panofsky-Wenzel theorem [2] is used in estimating the transverse emittance due to the multipole components of $E_{z}$.
\end{abstract}

\section{Introduction}

To produce high brightness low emittance electron beams necessary for X-ray FEL application such as the LCLS [3] we have developed a 1.6 cell emittance compensated S-band photocathode RF Gun that is designed to minimize emittance growth due to the dipole component of $E_{z}$. To accomplish this we have eliminated the side coupling into the half cell that is used in the original BNL RF gun design [4], which is designed to suppress the zero-mode. The coupling between the two cells was improved by an increase in the beam iris, which also increased the mode separation between the zero and $\pi$-modes. The coupling between the waveguide and full cell was studied using two different coupling schemes. The full cell was symmetrized to minimize emittance growth due to the $T M_{110}$ mode. The half cell length has been increased to provide more RF focusing. Resistive temperature control was elected over water cooling to facilitate design and minimize construction costs.

\section{Field Balance versus Mode Separation}

The full cell to half cell coupling is vital since side coupling was not used in this gun design. We further improved the technique of using mode separation for field balance tuning [5]. To measure the field balance versus mode separation it is necessary to measure $E_{z}$ on axis for the $\pi$-mode,

-Work supported by the Department of Energy, contracts DEAC03-76SFO0515 and DE-AC02-76CH00016 $f_{\pi}$ and $f_{0}$ for different full and half cell frequencies. For large difference in the full and half cell frequencies the $f_{\pi}$ and $f_{0}$ are essentially the independent cell frequencies, but when the cell frequencies are close to each other the coupling, which is frequency independent, separates the cell frequencies into the $f_{\pi}$ and $f_{0}[6]$. Field balance versus mode separation data is presented in figure 1. This data was taken using a $.472 \mathrm{~cm}$ diameter dielectric sphere and a self excited loop instead of a network analyzer which increased the accuracy of the frequency measurement down to $100 \mathrm{~Hz}$. The predictions of our equivalent circuit model,

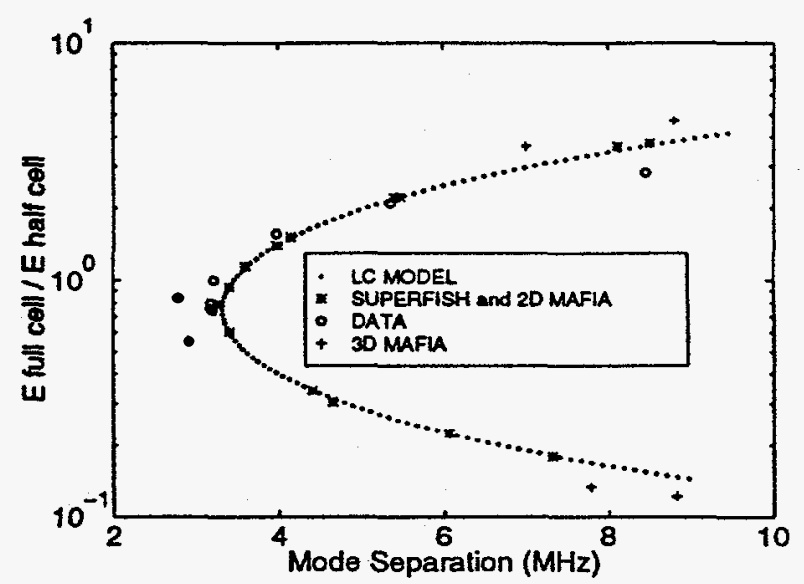

Figure. 1. Field Balance versus Mode Separation

2-D field solvers such as SUPERFISH [7] and the 3-D field solver MAFIA [8] are also shown in figure 1.

\section{Waveguide to Full Cell Coupling}

We have investigated two different types of waveguide to full cell coupling schemes. The $\theta$-coupling scheme was produced using a $.375^{\prime \prime}$ diameter cutting flute running $\pm .2268^{\prime \prime}$ center to center in the $x$ direction, the thickness of the coupling iris is $.0917^{\prime \prime}$. The $z$-coupling scheme was produced by cutting out a $.913^{\prime \prime} \times .650^{\prime \prime}$ rectangle with the long dimension in the $z$-direction, the thickness of the coupling iris was $.108^{\prime \prime}$. In the final gun design we have elected to use the $\theta$-coupling scheme since its dipole field, before symmetrization, is an order of magnitude less than the zcoupling scheme. We have measured the dipole field offset by a transverse disk pull in the waveguide plane and the 
multi-pole field components of the full cell by a needle rotation technique before and after symmetrizing the full cell for both schemes.

\section{Transverse Disk Pulls}

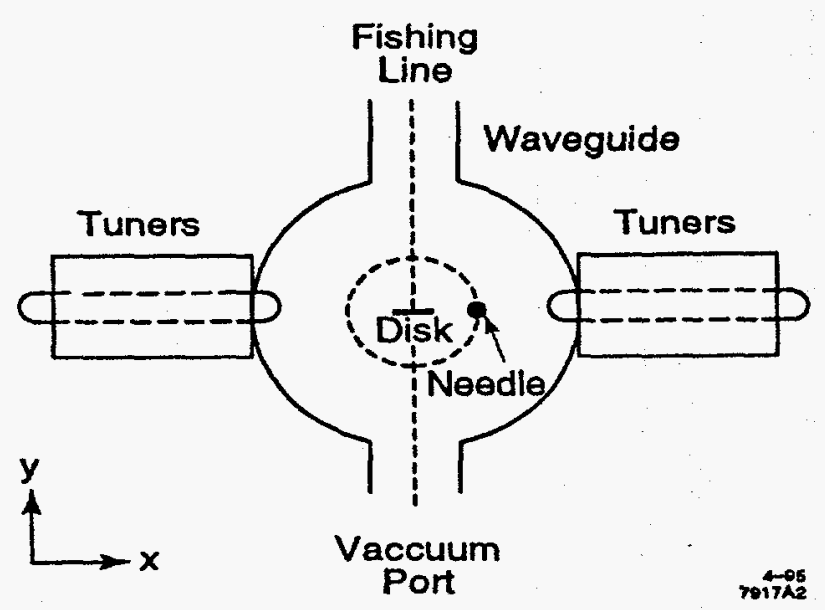

Figure. 2. Experimental Setups for Full Cell Disk Pull and Transverse View of Full Cell Needle Rotation

Figure 2 shows the experimental setup for our transverse disk pull, using a $6.35 \mathrm{~mm}$ diameter $0.30 \mathrm{~mm}$ thick $\mathrm{Cu}$ disk, used to measure $E_{z}\left(x_{0}, y\right)$. The dipole offset is directly measured by this technique assuming that $E_{z}$ can be modeled near the beam pipe axis by $E_{z}\left(x_{0}, y\right)=A+B(y-C)^{2}$. Experimental disk pull data is shown in figure 3 for the $\theta$ coupling scheme. The $\theta$-coupled dipole data along with waveguide to full cell coupling as a function of short position is shown in figure 4.

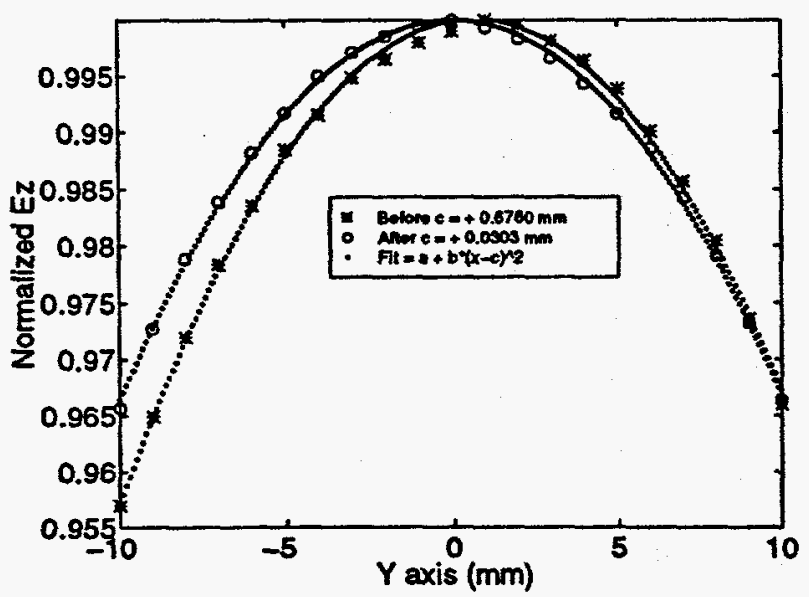

Figure. 3. 0-coupled Disk Pull Data Before and After Symmetrization

\section{Multi-pole Modes}

In previous measurements of the dipole field in an RF cavity, needles were pulled longitudinally offset from the beam axis [9] or were pulled transversely [10] to the beam axis. In our needle rotation technique a $1.27 \mathrm{~cm}$ long .635
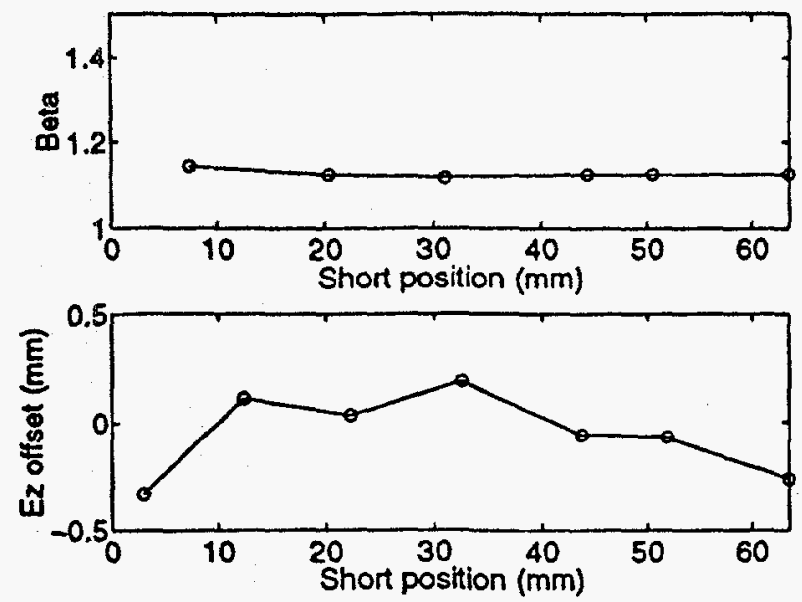

Figure. 4. $\quad$-coupled Full Cell to Waveguide Coupling $\beta$ and Dipole Offset versus Short Position

$\mathrm{mm}$ diameter needle is parallel to the beam axis and was rotated in the $\theta$ direction such that a cylinder was swept out, and thereby only perturbing the $E_{z}$. The experimental setup used for the needle rotation can be see in figure 2 and figure 5. Accurate positioning of the needle was accomplished by using a compound arrangement of translation and rotation stages. The translation stage allowed the positioning of the needle in the $r$ direction with respect to the beam pipe center. The rotation stage allowed the positioning of the needle in the $\theta$ direction with respect to a fiducial mark that we defined as the $x$-axis. It should be noted that the disk pull data is a subset of the needle rotation data. The full cell field, $E_{z}$, can be expressed as an infinite sum of modes represented by

$$
E_{z}=\sum_{m=0}^{\infty} \sum_{n=1}^{\infty} \sum_{l=0}^{\infty} A_{m n l} T M_{m n l}
$$

By measuring $E_{z}\left(r_{1}, \theta\right)$ as shown in figure 6 we can calculate the Fourier series coefficient $A_{m n l}$ of the infinite series, whose absolute values are shown in figure 7 . These coefficients are then used in the calculation of the emittance growth due to these multipole fields. Note that there is a large and changing (before and after) $x$-axis dipole field component, because the tuners were impossible to position precisely and in general were not symmetrical. Symmetrically feeding RF into the full cell would remove the phase asymmetry that exists in the cavity due to an asymmetric Poynting vector.

\section{Emittance Growth Estimation}

The change in the transverse normalized emittance is given by,

$$
\Delta \varepsilon_{y}=\sigma_{y} \frac{\Delta p_{y}}{m c}
$$

Using the Panofsky-Wenzel Theorem to find the change in momentum, which is in phase quadrature with $\frac{\partial E_{x}}{\partial y}$ we find that

$$
\frac{\Delta p_{y}}{m c}=\frac{e}{m c^{2}} \frac{\lambda}{2 \pi} \int_{0}^{L} \frac{\partial E_{z}}{\partial y} d z
$$




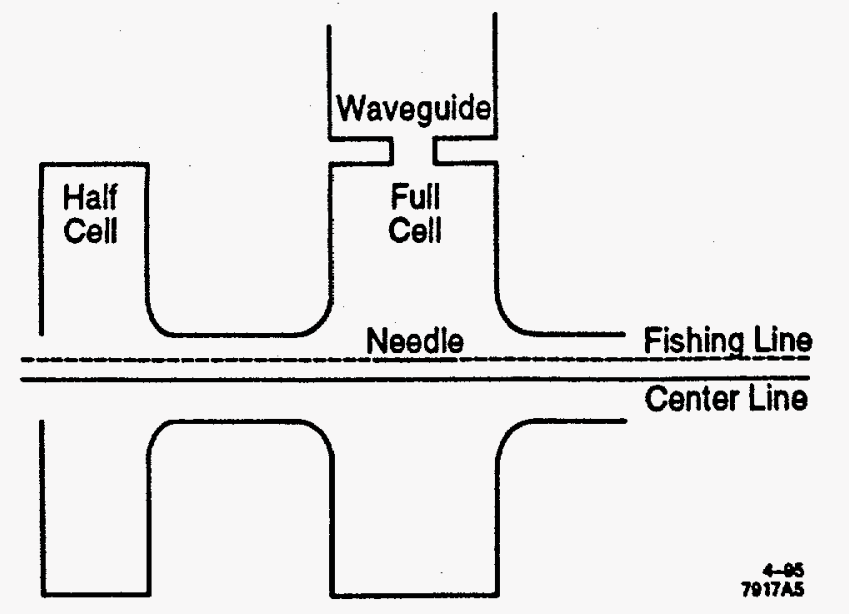

Figure. 5. Needle Rotation Experimental Setup

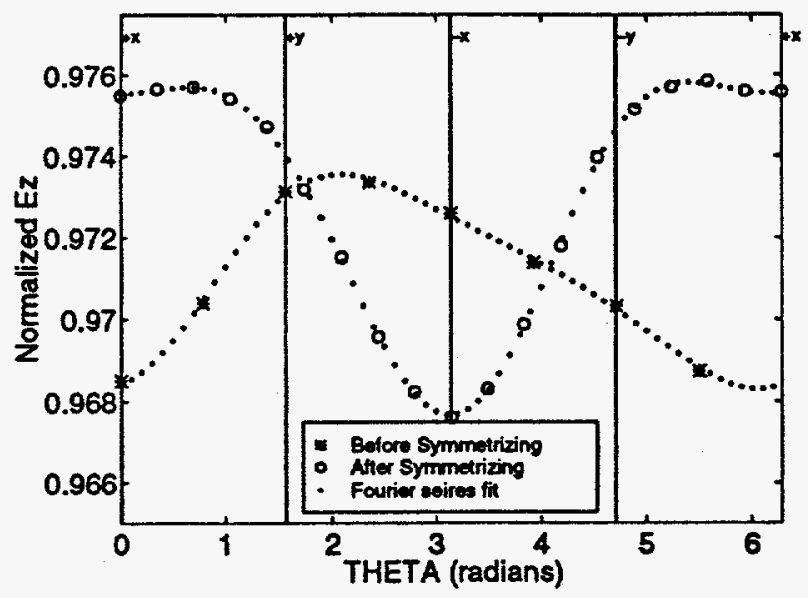

Figure. 6. $E_{z}\left(r_{1}, \theta\right)$ Before and After Symmetrization for the $\theta$-Coupling Scheme Using a Needle Rotation Technique

Therefore the change in the transverse emittance is

$$
\Delta \epsilon_{y}=\frac{e}{m c^{2}} \frac{\sigma_{z}}{2 \pi} \sigma_{y} \lambda \int_{0}^{L} \frac{\partial E_{z}}{\partial y} d z
$$

For the $T M_{110}$ and $T M_{210}$ type modes, $\frac{\partial E_{x}}{\partial y}$ is given by

$$
E_{z} \approx E_{m} \frac{r^{m}}{r_{1}^{m}} \sin \left(m \theta+\phi_{m}\right) \Rightarrow \frac{\partial E_{z}}{\partial y}=m \frac{E_{m}}{E_{0}} \frac{r^{m-1}}{r_{1}^{m}} E_{0}
$$

Therefore the change in the transverse emittance is

$$
\Delta \epsilon_{y} \approx \frac{e}{m c^{2}} \frac{\sigma_{z}}{2 \pi} \sigma_{y} \lambda n \frac{E_{m}}{E_{0}} \frac{r^{m-1}}{r_{1}^{m}} \int_{0}^{L} E_{0} d z
$$

Inserting the appropriate parameters from table $I$ of the electron bunch and the symmetrized RF gun that we are modeling. Where $r_{1}$ is the radius at which $E_{z}$ was measure at using the needle rotation technique and $\sigma_{y}$ is the beam size in the middle of the full cell. Assuming that $\frac{\partial E_{x}}{\partial y}$ can be approximated by a cosine like function of $z$ we find that the emittance growth in the symmetrized case is given by

$$
\Delta \epsilon_{y} \approx \begin{cases}0.1 \pi \mathrm{mm} \mathrm{mrad} & \text { Dipole mode } \\ 0.05 \pi \mathrm{mm} \text { mrad } & \text { Quadrapole mode }\end{cases}
$$

\begin{tabular}{|c|c|c|c|}
\hline$L$ & $.05 \mathrm{~m}$ & $\lambda$ & $10.5 \mathrm{~cm}$ \\
\hline$\sigma_{y}$ & $2.00 \mathrm{~mm}$ & $\sigma_{z}$ & $3 \mathrm{psec}$ \\
\hline$E_{0}$ & $100 \frac{M V}{m}$ & $r_{1}$ & $10.00 \mathrm{~mm}$ \\
\hline$m=1$ & Dipole & $m=2$ & Quadpole \\
\hline$\frac{E_{1}}{E_{0}}$ & 0.000094 & $\frac{E_{2}}{E_{0}}$ & 0.000084 \\
\hline
\end{tabular}

Table I

Electron bunch and RF gun cavity parameters

\section{Conclusions}

The dipole field components caused by the asymmetric RF coupling before and after symmetrizing the full cell were investigated. Data on the waveguide to full cell coupling and dipole offset versus short position was also taken. A needle rotation technique was used to measure the Fourier coefficient of the multipole field components of the full cell accelerating field. The dipole coefficient was seen to decrease by an order of magnitude after the full cell was symmetrized, with the proper positioning of the adjustable short that is located in the vacuum pumping port.

\section{References}

[1] L. C. Maier and J. C. Slater, Journal of Applied Physics, Vol.23, Num1 69 - 77, Jan 1952

[2] W. K. H. Panofsky and W. A. Wenzel, Rev. Sci. Instrum.,27,967 (1956)

[3] H. Winick et al NIM,A347,(1994),199-205

[4] K. Batchelor et al, Proc. of 1990 EPAC 541 - 543

[5] X.J. Wang et al, Proc. of 1993 PAC, $3000-3002$

[6] I. Wilson and H. Henke, CERN 89-09

[7] K. Halbach and R. F. Holsinger, Particle Accelerators 7, 213 (1976)

[8] T. Weiland et al. DESY Report, M-86-07 1986

[9] A. M. Vetter and R. P. Friedman, 1988 Linac Conf. Proc., CEBAF-Report-89-001, 58-60, June 1989

[10] Greg Loew private communication

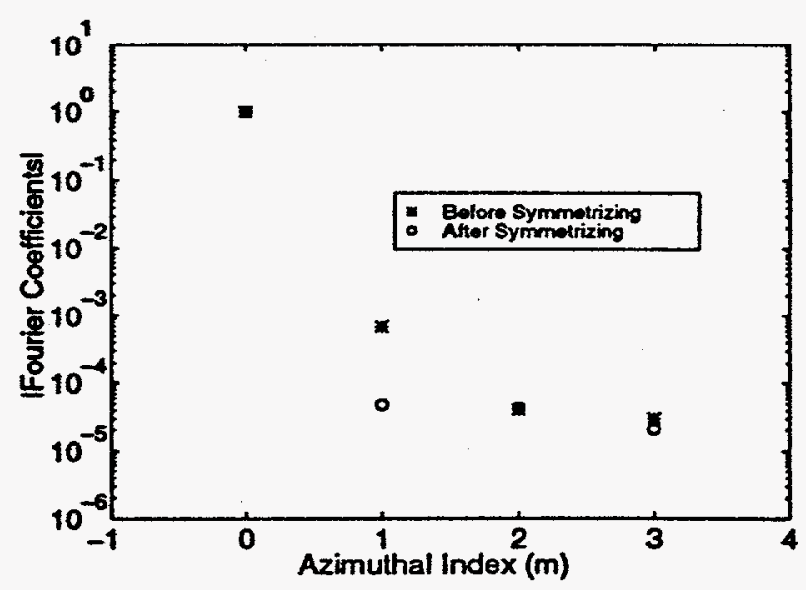

Figure. 7. $\theta$-coupled Fourier Coefficients Before and After Symmetrization 


\section{DISCLAIMER}

This report was prepared as an account of work sponsored by an agency of the United States Government. Neither the United States Government nor any agency thereof, nor any of their employees, makes any warranty, express or implied, or assumes any legal liability or responsibility for the accuracy, completeness, or usefulness of any information, apparatus, product, or process disclosed, or represents that its use would not infringe privateiy owned rights. Reference berein to any specific commercial product, process, or service by trade name, trademark, manufacturer, or otherwise does not necessarily constitute or imply its endorsement, recommendation, or favoring by the United States Government or any agency thereof. The views and opinions of authors expressed herein do not necessarily state or reflect those of the United States Government or any agency thereof. 


\section{DISCLAIMER}

Portions of this document may be illegible in electronic image products. Images are produced from the best available original document. 